\title{
Retraction Note to: TGF- $\beta$ Regulates Survivin to Affect Cell Cycle and the Expression of EGFR and MMP9 in Glioblastoma
}

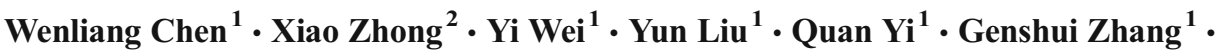 \\ Lishan $\mathrm{He}^{1} \cdot$ Fajiang Chen $^{1} \cdot$ Yingping Liu ${ }^{1} \cdot$ Jiandong Luo ${ }^{1,3}$
}

Published online: 6 May 2017

(C) Springer Science+Business Media New York 2017

Retraction Note to: Mol Neurobiol (2016) 53:1648-1653.

DOI 10.1007/s12035-015-9121-6

This article has been retracted at the request of the Editor-in-Chief and the Publisher per the Committee on Publication Ethics guidelines. There is strong reason to believe that the peer review process was compromised, and the authors have plagiarized parts from the following articles:

Anyan Liao, Ranran Shi, Yuliang Jiang, Suqing Tian, Panpan Li, Fuxi Song, Yalan Qu, Jinna Li, Haiqin Yun, and Xiangshan Yang, SDF-1/CXCR4 Axis Regulates Cell Cycle Progression and EpithelialMesenchymal Transition via Up-regulation of Survivin in Glioblastoma, Molecular Neurobiology,

The online version of the original article can be found at http://dx.doi.org/ 10.1007/s12035-015-9121-6

Jiandong Luo

luojiandong@gzhmu.edu.cn

1 Department of Pharmacology, Guangzhou Medical University, Guangzhou 510182, People's Republic of China

2 Department of Paediatrics, the Affiliated Xiaolan People's Hospital of Southern Medical University, Guangdong Zhongshan 528415, China

3 Guangzhou Institute of Cardiovascular Disease, Guangzhou Key Laboratory of Cardiovascular Disease, the Second Affiliated Hospital, Guangzhou Medical University, Guangzhou, People's Republic of China
January 2016, Volume 53, Issue 1, pp. 210-215, DOI: 10. 1007/s12035-014-9006-0; Received: 1 November 2014

Peng Yang, Gang Wang, Hongjun Huo, Qiang Li, Yan Zhao, and Yuanhang Liu, SDF-1/CXCR4 signaling upregulates survivin to regulate human sacral chondrosarcoma cell cycle and epithelial-mesenchymal transition via ERK and PI3K/AKT pathway, Medical Oncology, January 2015, 32:377, DOI: 10.1007/s12032014-0377-x; Received: 13 November 2014

As such, the validity of the content of this article cannot be verified. 